\title{
A Case Report and Literature Review of Pediatric Joint Hyperactivity Syndrome
}

\author{
Gaohong $\mathrm{Wu}^{1} \cdot$ Xueping $\mathrm{Zhu}^{1}$
}

Accepted: 25 January 2019 / Published online: 6 February 2019

(C) The Author(s) 2019

Joint hypermobility syndrome (JHS) is a hereditary connective tissue disease characterized by excessive joint activity, skin fragility, arthrodynia of limbs, fatigue, autonomic dysfunction, and gastrointestinal dysperistalsis [1]. There are few domestic reports. In order to improve the understanding of JHS clinical diagnosis and treatment, and to reduce misdiagnosis and missed diagnosis, a typical JHS report in our hospital is reported as follows.

\section{Case Report}

The patient was male, 13 years old, and was admitted to the hospital due to "polyarthralgia for more than 3 months." There was pain in both feet without obvious inducement 3 months ago, especially when carrying weight. Later, knee, hip, palm, wrist, elbow, shoulder, and lower back pain gradually appeared. The patient was physically healthy in the past, but his mother and cousins had JHS. The patient's mind was clear and with normal reaction when being admitted to the hospital. The skin and mucous membranes of the whole body did not have bleeding spots, there was no neck rigidity, the throat was not red, the tonsils were not big, and lung auscultation determined breath sounds are rough. There were regular heart rhythm, powerful heart sound, and no murmur in the auscultation area of each valve. The abdomen was soft; the liver and spleen were not palpable under the ribs; hyperextend the elbow by more than $10^{\circ}$; passively dorsiflex the fifth metacarpophalangeal joint by more than $90^{\circ}$; oppose the

This article is part of the Topical Collection on Medicine

Xueping Zhu

zhuxueping4637@hotmail.com

Gaohong Wu

1217555215@qq.com

1 Department of Neonatology, Children's Hospital of Soochow University, Suzhou 215025, Jiangsu, China thumb to the volar aspect of the ipsilateral forearm, and the lower limbs are restricted in activity, and the double " 4 " sign was positive. The limb endings were warm, and the nervous system was not abnormal. Related examinations of admission: blood routine: white blood cells $4.48 \times 10^{9} / \mathrm{L}$, red blood cells $4.97 \times 10^{12} / \mathrm{L}$, lymphocytes $51.5 \%$, basophils $1.3 \%$, eosinophils $7.3 \%$, hemoglobin $150 \mathrm{~g} / \mathrm{L}$, total platelets $178 \times 10^{9} / \mathrm{L}$, neutrophils $32.1 \%$, neutrophil absolute count $1.44 \times 10^{9} / \mathrm{L}$; blood gas analysis + electrolyte: standard bicarbonate (SB) $23.9 \mathrm{mmol} / \mathrm{L}$, carbon dioxide partial pressure $\left(\mathrm{PCO}_{2}\right)$ $40.5 \mathrm{mmHg}$, calcium $0.93 \mathrm{mmol} / \mathrm{L}$, corrected calcium (pH 7.4) $0.93 \mathrm{mmol} / \mathrm{L}$, blood $\mathrm{pH} 7.387$; complete urine protein test: $N$-acetylglucosaminidase $24.89 \mathrm{U} / \mathrm{L}$, urine immunoglobulin G $28.92 \mathrm{mg} / \mathrm{L}$, urine microalbumin $190.9 \mathrm{mg} / \mathrm{L}$, urinary transferrin $7.25 \mathrm{mg} / \mathrm{L}$. There is no obvious abnormality in urine routine, fecal routine + occult blood test, coagulation routine (D dimer), PCT, ESR, tuberculosis antibody, Mycoplasma pneumoniae antibody, anti-cyclic citrullinated peptide antibody, anti-chain $\mathrm{O}$, rheumatoid factor, human leukocyte antigen, lymphocyte subset, biochemical complete set, humoral immunity, ANCA, anti-keratin antibody, antiphosphoester antibody, autoantibody screening, three-item test before blood transfusion, antigens and antibodies of hepatitis B (quantitative), and bone marrow smear. No genetically relevant mutations were found in the genetic tests. There were no abnormalities upon chest radiograph, supercardiogram, and magnetic resonance (bilateral knee, ankle joint, hip joint). The child had a family history of joint hyperactivity and was admitted to the hospital with joint pain of the limbs. The examination showed excessive joint activity (Fig. 1). According to the Beighton score [] (Table 1), the cscore of the child is 6. According to Brighton standard [2] (Table 2), the child meets two major criteria and one minor criterion, so the diagnosis of joint hyperactivity syndrome is clear. After 7 days of treatment with ibuprofen, total glucosides of paeony capsules, vitamins A and D, and five vitamins and calcium gluconate, from 26 November 2017, the patient's joint pain was relieved, and was discharged on 3 December 2017. He has been 
Fig. 1 Special signs of the child: a finger side passive back flexion parallel to forearm extension side; b elbow joint extension $>10^{\circ}$; c passive motion of the thumb can touch the flexion of the forearm

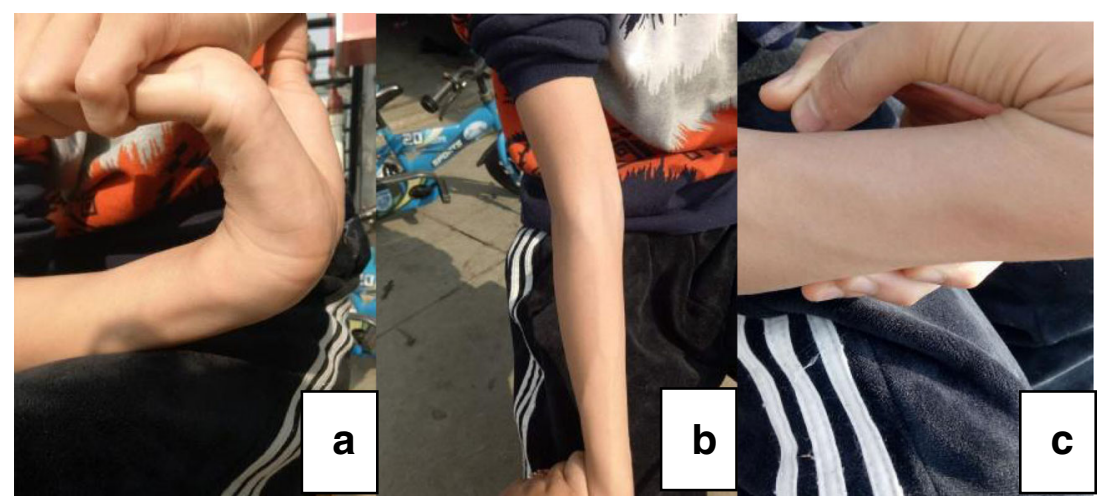

followed for nearly half a year. After family education, psychological counseling, and appropriate medication, the joint pain was significantly relieved according to the 3 June 2018 telephone follow-up and the patient has resumed school now.

\section{Discussion}

JHS is also known as arthrochalasis. The exact incidence rate is unknown. There are many reports abroad. It was first reported by Kirk et al. [3], who considered it to be a familial and hereditary connective tissue disease, mainly found in children. The main clinical manifestations of this disease are as follows: (1) skeletal muscle lesions: JHS, joint pain, joint dislocation, poor body balance, horse fan-shaped signs, etc.; (2) skin lesions: high ductility, crisp, incident contusion, obvious wrinkle, etc.; (3) gastrointestinal symptoms: abdominal distension, early satiety, alternating diarrhea and constipation, nausea, vomiting, and even gastroesophageal reflux, esophageal hiatus hernia, etc.; (4) bladder dysfunction: frequent and urgent urination, urinary incontinence, etc.; (5) other: chronic fatigue, depression, fear, palpitations, chest pain, approximate syncope or syncope, postural hypotension, varicose veins, eye deformities, and so on. There are generally no special findings in laboratory examination of this disease, and the diagnosis mainly depends on medical history and physical examination. At present, the Beighton score and the Brighton standard based on it are often used, and clinical geneticists can help in identification or exclude other hereditary connective tissue diseases. On the differential diagnosis, this disease is mainly differentiated from MarFan syndrome, Ehlers-Danlos syndrome, juvenile idiopathic arthritis, rheumatic fever, and homocystinuria. The differential diagnosis analysis of this patient is as follows: (1) MarFan syndrome is an autosomal dominant hereditary disease, its common symptoms are tall figure, acromacria, congenital cardiovascular malformation, lens translocation, etc. The main hazard is cardiovascular lesion, especially aortic aneurysms, and can be diagnosed according to the clinical manifestations of three main signs in bone, eye, and cardiovascular changes, and family history. The simplest method of diagnosing this disease is echocardiography, and further confirmation requires MRI. The child was mainly characterized by polyarthralgia and excessive joint activity. There were no manifestations of MarFan syndrome such as tall figure and acromacria. There was no abnormality in laboratory tests of cardiac ultrasonography and magnetic resonance; therefore, this disease can be ruled out. (2) Ehlers-Danlos syndrome is a disease that has a genetic predisposition of affecting connective tissue and is associated with defects in collagen metabolism. The skin of the affected part is stretched excessively and upon touching is soft, like a velvety feeling. Due to excessive skin stretching, incidents form wounds, blood vessels are brittle and easy to rupture, and skin bruises; it often presents joint dislocation, tube wall tumor by cardiovascular and gastrointestinal tract enlargement, gastrointestinal diverticulum, bladder diverticulum or ruptured perforation, etc. This disease is more common in premature infants, and can be diagnosed according to the features of the increase in excessive skin elasticity and joint activity, increased skin and blood vessel fragility, and the emergence of pseudo-tumor after trauma. The child was a 13-year-old boy who showed only joint pain and excessive

Table 1 Beighton score

\begin{tabular}{llc}
\hline Special ability & Left & Right \\
\hline Elbow joint extension $>10^{\circ}$ & 1 & 1 \\
Knee extension $>10^{\circ}$ & 1 & 1 \\
Finger side passive back flexion parallel to forearm extension side & 1 & 1 \\
Passive motion of the thumb can touch the flexion of the forearm & 1 & 1 \\
Keep your knees straight, bend your knees forward and touch the floor & & 1 \\
\hline
\end{tabular}

A score greater than or equal to 4 points may be JHS 
Table 2 Brighton standard

\begin{tabular}{|c|c|}
\hline Main indicators & Secondary indicators \\
\hline $\begin{array}{l}\text { Beighton score is greater than or equal } \\
\text { to } 4 \text { points (current or previous) }\end{array}$ & If the age is over 50 , the Beighton score is 1,2 , or 3 \\
\hline \multirow[t]{7}{*}{$\begin{array}{l}\text { Greater than or equal to } 4 \text { joints pain } \\
\text { for more than } 3 \text { months }\end{array}$} & $\begin{array}{l}1 \text { to } 3 \text { joint pain more than } 3 \text { months, } \\
\text { low back pain } 3 \text { months, or spine lesions }\end{array}$ \\
\hline & Greater than or equal to 1 joint dislocation or subluxation \\
\hline & $\begin{array}{l}\text { In } 3 \text { or more locations of soft tissue rheumatism } \\
\text { (such as epicondylitis, tenosynovitis, bursitis) }\end{array}$ \\
\hline & Somatotype of Marfan syndrome \\
\hline & Abnormal skin (such as atrophy high ductility crisp or paper scars) \\
\hline & Abnormal eye (such as blepharoptosis myopia) \\
\hline & Varicose hernia prolapse of the uterus or rectum \\
\hline
\end{tabular}

JHS can be diagnosed by meeting two of the above main criteria, one main criterion plus two secondary criteria or four secondary criteria

activity, no skin elasticity, overstretching, and other organ damage; therefore, this disease can be ruled out. (3) Juvenile idiopathic arthritis refers to arthritis lasting for over 6 weeks of unknown courses for children over 16 years of age. The age and duration of the disease were consistent with the condition of juvenile idiopathic arthritis, but there was no arthritis characterization such as joint swelling, joint fluid accumulation, joint activity limitation, and local joint fever. It is not very unlikely that the disease is considered. (4) Rheumatic fever is a systemic connective tissue immune inflammatory disease caused by Group A Streptococcus infection, mainly invading the heart, joints, skin, serosa, and central nervous system and lungs, kidneys, and other internal organs. This disease mainly relies on clinical manifestations; the current diagnosis is still under the 1992 revised Jones standard. In this case, there was no history of streptococcal infection, only joint pain and excessive activity, no special findings upon laboratory tests, and did not meet the diagnostic criteria for rheumatic fever. (5) Homocystinuria is an inheritance disease caused by the lack of cystathionine synthetase during the process of methionine dissimilation and is associated with mental retardation, developmental disorders, abnormal lens position, limb rigidity, thinning hair, abnormal cardiovascular system, etc. It can be diagnosed according to clinical symptoms such as typical skeletal malformations, lens shift, mental retardation and mental decline, vascular occlusion with thrombosis or embolism, plasma cysteine and methionine increase, etc. Although the hematuria genetic metabolism screening was not conducted on the child, based on its clinical manifestations, laboratory results, and the proper treatment effect of JHS, this disease is not considered. In this case, the patient was admitted to the hospital with polyarthralgia for more than 3 months. There was a history of excessive joint activity in the family. The over-elbow joint was overextended by more than $10^{\circ}$. The finger side by side passive back is parallel with the forearm extension side, the passive movement of the thumb can touch the flexion of the forearm, and the lower limbs are restricted in activity, and the double " 4 " sign was positive. The Beighton score is
6 points. The Brighton standard meets 2 major criteria and 1 minor criterion, so the diagnosis is clear. However, there is still a lack of research on relevant specific laboratory indicators.

The disease is benign, and generally does not affect the normal growth and development of children. Condition joint relaxation phenomenon will gradually improve in most of the children with age; the key is to conduct the diagnosis early, and to avoid misdiagnosis and missed diagnosis. The treatment should be personalized according to the patient's clinical manifestations; pain management should be in a multidisciplinary approach, for example, oral aspirin, ibuprofen, etc. when the joint pain is obvious, and appropriate rest; paying attention to physical therapy, clinical-related occupational therapy, and appropriate physical training (Pilates training, Tai Chi, etc.) can improve joint stability and strength, avoid injuries, and help patients overcome life difficulties. In addition, JHS patients and their families should receive education on the nature of the disease to relieve anxiety and nervousness [4-8]. The child had obvious joint pain and limited mobility, and he was relieved after intervention with ibuprofen and physical therapy. Since the onset of the disease, the child's mood has been low, with lazy words and less movement, and their parents were also emotionally anxious and nervous, invisibly expanding the severity of the disease. As medical staff, we should strengthen our understanding of the disease, educate and communicate with patients and their families in a timely manner, and pay attention to psychological treatment programs. The above treatments for this disease are based on previous experience and the summary of the characteristics of this child. There is still a lack of randomized controlled trials to verify the efficacy of the disease [9]. Therefore, it needs in-depth basic research and randomized, multicenter, large-sample clinical studies, especially long-term prognosis follow-up and assessment to provide a basis for establishing clinical guidelines. 


\section{Compliance with Ethical Standards}

Conflict of Interests The authors declare that they have no conflict of interest.

Ethical Approval All procedures performed in studies involving human participants were in accordance with the ethical standards of the institutional.

Informed Consent Informed consent was obtained from all individual participants included in the study.

Open Access This article is distributed under the terms of the Creative Commons Attribution 4.0 International License (http:// creativecommons.org/licenses/by/4.0/), which permits unrestricted use, distribution, and reproduction in any medium, provided you give appropriate credit to the original author(s) and the source, provide a link to the Creative Commons license, and indicate if changes were made.

Publisher's Note Springer Nature remains neutral with regard to jurisdictional claims in published maps and institutional affiliations.

\section{References}

1. Syx D, De IW, Rombaut L, et al. Hypermobility, the Ehlers-Danlos syndromes and chronic pain.[J]. Clin Exp Rheumatol. 2017;35 Suppl 107(5):116.
2. Grahame R, Bird HA, Child A. The revised (Brighton 1998) criteria for the diagnosis of benign joint hypermobility syndrome (BJHS). J Rheumatol. 2000;27(7):1777-9.

3. Kirk JA, Ansell BM, Bywaters EG. The hypermobility syndrome. Musculoskeletal complaints associated with generalized joint hypermobility.[J]. Ann Rheum Dis. 1967;26(5):419.

4. Ferrell WR, Tennant N, Sturrock RD, Ashton L, Creed G, Brydson $\mathrm{G}$, et al. Amelioration of symptoms by enhancement of proprioception in patients with joint hypermobility syndrome. Arthritis Rheum. 2004;50(10):3323-8.

5. Simmonds JV, Keer RJ. Hypermobility and the hypermobility syndrome. Man Ther. 2007;12(4):298-309.

6. Simmonds JV, Keer RJ. Hypermobility and the hypermobility syndrome, part 2: assessment and management of hypermobility syndrome: illustrated via case studies. Man Ther. 2008;13(2):1-11.

7. Celletti C, Castori M, Torre GL, et al. Evaluation of kinesiophobia and its correlations with pain and fatigue in joint hypermobility syndrome/Ehlers-Danlos syndrome hypermobility type. Biomed Res Int. 2013;2013(1):580460.

8. Hakim AJ, Ashton S. Undiagnosed joint hypermobility syndrome patients have poorer outcome than peers following chronic back pain rehabilitation. Rheumatology. 2005;44(suppl 1):i106.

9. Smith TO, Bacon H, Jerman E, et al. Physiotherapy and occupational therapy interventions for people with benign joint hypermobility syndrome: a systematic review of clinical trials [J]. Disabil Rehabil. 2014;36(10):797-803.s. 